\title{
Analytical Modelling of IEEE 802.15.4 for Multi-hop Networks with Heterogeneous Traffic and Hidden Terminals
}

\author{
Piergiuseppe Di Marco, Pangun Park, Carlo Fischione, Karl Henrik Johansson
}

\begin{abstract}
IEEE 802.15.4 multi-hop wireless networks are an important communication infrastructure for many applications, including industrial control, home automation, and smart grids. Existing analysis of the IEEE 802.15.4 medium access control (MAC) protocol are often based on assumptions of homogeneous traffic and ideal carrier sensing, which are far from the reality when predicting performance for multi-hop networks. In this paper, a generalized analysis of the unslotted IEEE 802.15.4 MAC is presented. The model considers heterogeneous traffic and hidden terminals due to limited carrier sensing capabilities, and allows us to investigate jointly IEEE 802.15.4 MAC and routing algorithms. The analysis is validated via Monte Carlo simulations, which show that routing over multi-hop networks is significantly influenced by the IEEE 802.15.4 MAC performance. Routing decisions based on packet loss probability may lead to an unbalanced distribution of the traffic load across paths, thus motivating the need of a joint optimization of routing and MAC.
\end{abstract}

\section{INTRODUCTION}

IEEE 802.15.4 is becoming the dominant communication standard for wireless low power and low data rate networks [1]. There is a wide range of applications for IEEE 802.15.4 in industrial monitoring, building automation, health care, environmental monitoring, urban sensor networks, etc. Routing information over multi-hop paths is a fundamental networking service for many of these applications [2]. There is not yet a definite solution for routing, but a lively research activity and standardization effort are ongoing by the Routing over Low Power and Lossy Networks (ROLL) working group of the Internet Engineering Task Force [2], which is defining a routing protocol on top of the physical layer and medium access control (MAC) of IEEE 802.15.4. We believe that an accurate mathematical modelling of IEEE 802.15.4 MAC for multi-hop topologies is instrumental to understand the fundamental performance limitations of these networks.

For single-hop star networks, many papers in the literature have proposed models for capturing the behavior of the IEEE 802.15.4 MAC with either saturated or unsaturated traffic, with or without acknowledgements and retransmissions (see, e.g., [3] - [5]). These studies are based on extensions of the Markov chain model originally proposed by Bianchi [6] for the IEEE 802.11 protocol. In all these contributions, traffic is assumed to be homogeneous, both in the saturated and in the

The authors are with the ACCESS Linnaeus Center, Electrical Engineering, Royal Institute of Technology, Stockholm, Sweden. E-mail: \{pidm|pgpark|carlofi|kallej\}@ee.kth.se.

The authors acknowledge the support the Swedish Foundation for Strategic Research, the Swedish Research Council, the Swedish Governmental Agency for Innovation Systems, and the EU project FeedNetBack. unsaturated scenarios. This assumption is a major limitation in at least three important situations:

1) In single-hop networks, nodes may have different traffic generation rates as a result of different services that they have to provide.

2) In multi-hop networks, the traffic load varies according to the routing along the paths. Some node may experience a heavier cross traffic, thus transmitting more packets than nodes that are traversed by fewer routing paths. It follows that the traffic is not homogeneous, regardless the fact that nodes may generate their own packets at the same rate.

3) In networks with hidden terminals, the traffic of the network perceived by the nodes is different from node to node, even though every node may generate the same traffic. This is due to that some nodes may not perceive other nodes transmitting.

In the scenarios mentioned above, existing analytical studies of the IEEE 802.15.4 MAC are not sufficient. Some studies for IEEE 802.11 and for IEEE 802.15.4 with heterogenous traffic conditions can be found in the literature ( [7] - [9]), where two traffic classes have been considered. For the case of single-hop IEEE 802.11 star network topologies and saturated traffic, the effects of hidden terminals have been studied in [10] and [11]. In [12], multi-hop communication is modelled for IEEE 802.11 networks but with a single traffic pattern. In [13], the work of [12] has been extended to multiple non-saturated flows. However, we note that these models can not be directly applied to IEEE 802.15.4 networks due to the different access mechanism of IEEE 802.11.

In this paper we propose two original contributions. First, we give a comprehensive Markov chain model for the unslotted IEEE 802.15.4 MAC in the presence of both heterogeneous traffic and limited carrier sensing range. We show how existing studies from the literature we have mentioned above are inadequate in the proposed scenarios. Second, we provide an analysis of multi-hop networks, giving a framework to study the complex interaction between IEEE 802.15.4 MAC and routing layers. We show that the IEEE 802.15.4 MAC reliability may influence substantially the routing and affect negatively the routing decisions. Therefore, we believe that this study may have impact on the ongoing standardization process for ROLL [2].

The remainder of the paper is organized as follows. In Section II, we introduce the system model. In Section 【I, we derive a new analytical model of IEEE 802.15.4 MAC for multi-hop networks with heterogeneous traffic, hidden 
terminals, and limited carrier sensing range. The accuracy of the model is evaluated by extensive Monte Carlo simulations in Section IV] Section $\mathrm{V}$ concludes the paper and prospects our future work.

\section{SySTEM MODEL}

Consider a network of $N$ nodes that use the unslotted IEEE 802.15.4 MAC. We focus on this MAC modality because of the relevance for the ROLL standardization [2]. In the following, we illustrate the system model by considering two topologies, as reported in Fig. 1. However, the analytical results that we derive in the following are general and do not depend on the specific topology assumed in the figure.

Topology a) refers to a single-hop (star) topology where nodes forward their packets with single-hop communication to the root node $V_{0}$. We denote by $l$ the link between node $V_{l}$ and $V_{0}$. Topology b) is an example of multi-hop network in which nodes forward traffic according to the uplink routing policy to $V_{0}$.

For every node $V_{l}$, we define a neighborhood set $\Omega_{l}$, which contains all the nodes in the carrier sensing range of $V_{l}$ (delimited by dashed lines in Fig. 11. The carrier sensing rage is the set of nodes that can be heard by a node while performing the IEEE 802.15.4 clear channel assessment (CCA). We denote with $\left|\Omega_{l}\right|$ the cardinality of $\Omega_{l}$. For each node $V_{l}$, we define a parent set $\Gamma_{l} \subset \Omega_{l}$, which contains all nodes that may be nexthop nodes of $V_{l}$, and children set $\Delta_{l} \subset \Omega_{l}$, which contains all nodes that have $V_{l}$ as next-hop node. Let $\Omega_{j \backslash l}=\Omega_{j}-\Omega_{l} \cap \Omega_{j}$ be the hidden node set, namely all nodes that are in the carrier sensing range of the receiver $V_{j}$, but that do not belong to the carrier sensing range of the transmitter $V_{l}$.

We assume that the channel is symmetric, so that if $V_{k} \in \Omega_{l}$, then $V_{l} \in \Omega_{k}$, which is natural when transmitting and receiving over similar frequencies. Furthermore, we assume that the communication range is equal to the carrier sensing range of a node. Such assumptions are widely adopted, e.g., [3] - [12].

We consider the routing specifications by ROLL [2]. Coherently with the standard, the root node generates a destinationoriented directed acyclic graph (DODAG). The main property of a DODAG is that all edges are oriented in such a way that no cycles exist. Directional routes in the network are indicated by arrows in Fig. 1 In the following section, we introduce the model for multi-hop unslotted IEEE 802.15.4 MAC and we derive the basic relations with the routing policy.

\section{Multi-Hop Unslotted IEEE 802.15.4 MAC}

In this section, a generalized model of a heterogeneous unslotted IEEE 802.15.4 network is proposed. The analysis aims at deriving the probability of successful packe 11 reception. We start the analysis by considering a single-hop case, and then we generalize the analysis to the multi-hop case.

\section{A. Single-Hop Network Model}

The single-hop analysis is based on the model for slotted IEEE 802.15.4 random access presented in [5], which accounts for retry limits, acknowledgements, and unsaturated traffic.

\footnotetext{
${ }^{1}$ Throughout this paper, we denote packets the MAC protocol data units.
}

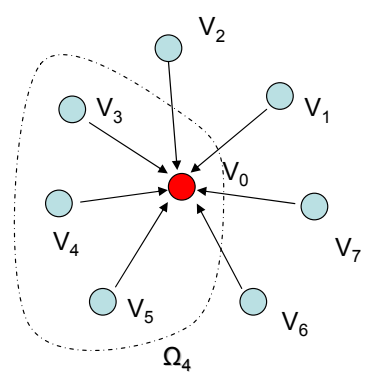

a) Single-hop topology

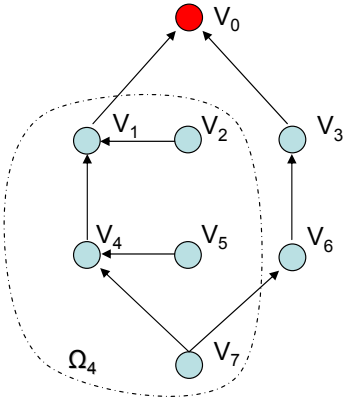

b) Multi-hop topology
Fig. 1. Example of a single-hop topology (left) and a multi-hop topology (right) IEEE 802.15.4 network. The dashed area $\Omega_{4}$ delimits the carrier sensing range of node $V_{4}$, namely the largest set of nodes that can be heard by $V_{4}$ while doing the IEEE 802.15.4 clear channel assessment.

We extend substantially the analysis to realistic characteristics of the unslotted mechanism, namely single clear channel assessment (CCA) and unsynchronized slots, and we include limited carrier sensing range and heterogeneous traffic.

The generation of unsaturated traffic at node $V_{l}$ is modelled by the packet generation probability $q_{l}$. In particular, $q_{l}$ is the probability of generating a new packet in each time unit, when the node is in idle state. We measure traffic as

$$
\lambda_{l}=\frac{q_{l}}{\text { aUnitBackoffPeriod }} \quad(\mathrm{pkt} / \mathrm{s}),
$$

where aUnitBackoffPeriod is the duration of a time unit. We remark that $\lambda_{l}$ is an upper bound for the actual node traffic rate, because it does not consider the generation of packets during the random backoff and during the packet transmission time. However, it has the advantage of a simple relation with the Markov chain. The approximation is accurate for practical applications, particularly as the traffic reduces [5].

Let $\alpha_{l}$ be the probability of busy channel assessment, and $P_{\text {coll }, l}$ be the packet collision probability experienced in the link $l$. We denote the IEEE 802.15.4 MAC parameters by $W_{0}=2^{\text {macMinBE }}, m_{0}=\operatorname{macMinBE}, m_{b}=\operatorname{macMaxBE}, m=$ macMaxCSMABackoffs, $n=$ macMaxFrameRetries (see [1] for details on the IEEE 802.15.4 standard).

Consider the Markov chain of Fig. 2, which models the unslotted IEEE 802.15.4 with retry limits, acknowledgements, and unsaturated traffic for the transmitting node in each link. We first derive the probability $\tau_{l}$ that node $V_{l}$ attempts the clear channel assessment, by solving the Markov chain associated to link $l$. Second, we couple the per-link Markov chains to obtain a set of equations to be able to derive the network operating point. Details follow in the sequel.

Let $c_{l}(t)$ be the stochastic process representing the counter for random backoff and channel occupation for packet and acknowledgement transmission, associated to the transmitting node in link $l$. During the transmission stage, $L+L_{\text {ack }}$ slots are accounted, where $L$ and $L_{\text {ack }}$ denote the packet transmission and acknowledgment transmission duration measured in slot:2. Let $s_{l}(t)$ be the stochastic process representing the delay line stages that counts the number of times the channel is sensed busy before packet transmission $\left(s_{l}(t) \in\{0, \cdots, m\}\right)$, or the

\footnotetext{
${ }^{2} \mathrm{We}$ assume that this duration is an integer number of slots.
} 


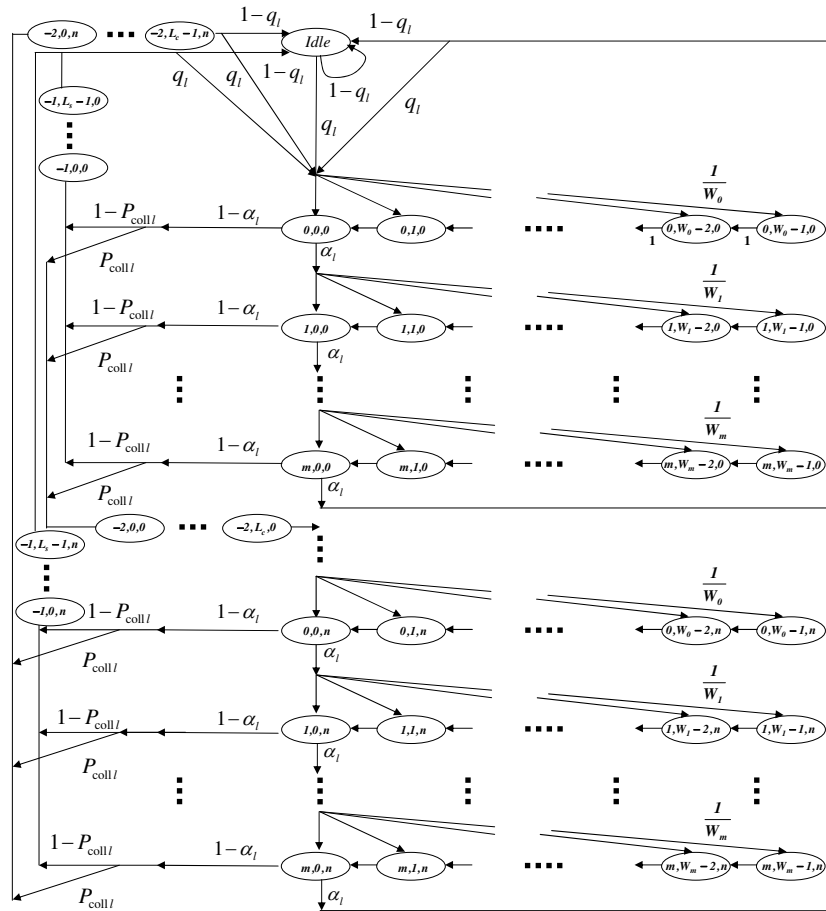

Fig. 2. Markov chain model of the CSMA/CA algorithm of transmitting node in link $l$ for unslotted IEEE 802.15.4 MAC.

transmission stage $\left(s_{l}(t)=-1\right)$ at time $t$. Let also $r_{l}(t)$ be the stochastic process representing the retransmission counter.

We assume that the probability to start sensing for each node is independent of the number of retransmissions suffered. With this assumption, $\left\{s_{l}(t), c_{l}(t), r_{l}(t)\right\}$ is the three-dimensional Markov chain of Fig. 2 with transition probabilities

$$
\begin{aligned}
& \operatorname{Pr}[i, k, j \mid i, k+1, j]=1, \text { for } k \geq 0, \\
& \operatorname{Pr}[i, k, j \mid i-1,0, j]=\frac{\alpha_{l}}{W_{i}}, \text { for } i \leq m, \\
& \operatorname{Pr}[0, k, j \mid i, 0, j-1]=\frac{\left(1-\alpha_{l}\right) P_{\text {coll }, l}}{W_{0}}, \text { for } j \leq n, \\
& \operatorname{Pr}[\text { idle } \mid m, 0, j]=\left(1-q_{l}\right) \alpha_{l}, \text { for } j<n, \\
& \operatorname{Pr}[\text { idle } \mid i, 0, n]=\left(1-q_{l}\right)\left(1-\alpha_{l}\right), \text { for } i<m, \\
& \operatorname{Pr}[\text { idle } \mid m, 0, n]=q_{l}, \\
& \operatorname{Pr}[0, k, 0 \mid \text { idle }]=\frac{q_{l}}{W_{0}}, \text { for } k \leq W_{0}-1 .
\end{aligned}
$$

Eq. (1) is the decrement of backoff counter, which happens with probability 1. Eq. (2) represents the probability of finding busy channel in CCA and of choosing a state uniformly in the next backoff stage. Eq. (3) gives the unsuccessful transmission probability after finding a clear channel, and a node selects uniformly a state in the next retransmission stage. Eqs. (4) and (5) represent the probability of going back to the idle stage due to the channel access failure and retry limits, respectively. Eq. (6) is the probability of going back to the idle stage at backoff counter $m$ and retransmission stage $n$, as function of the traffic $q_{l}$. Eq. (7) models the probability of going back to the first backoff stage from the idle stage.

Let $b_{i, k, j}^{(l)}=\lim _{t \rightarrow \infty} \operatorname{Pr}\left[s_{l}(t)=i, c_{l}(t)=k, r_{l}(t)=j\right], i \in$ $(-2, m), k \in\left(0, \max \left(W_{i}-1, L_{s}-1, L_{c}-1\right)\right), j \in(0, n)$ be the stationary distribution of the Markov chain where $L_{s}, L_{c}$ are the time periods for successful transmission and packet collision, respectively.

By considering Eqs. (1) - (7), the normalization condition for the stationary distribution, and by assuming for simplicity that $m \leq m_{b}-m_{0}$, we obtain the expression for $b_{0,0,0}^{(l)}$ :

$$
\begin{aligned}
b_{0,0,0}^{(l)}= & {\left[\frac{1}{2}\left(\frac{1-\left(2 \alpha_{l}\right)^{m+1}}{1-2 \alpha_{l}} W_{0}+\frac{1-\alpha_{l}^{m+1}}{1-\alpha_{l}}\right) \frac{1-y_{l}^{n+1}}{1-y_{l}}\right.} \\
& +\left(L_{s}\left(1-P_{\mathrm{coll}, l}\right)+L_{c} P_{\mathrm{coll}, l}\right)\left(1-\alpha^{m+1}\right) \frac{1-y_{l}^{n+1}}{1-y_{l}} \\
& +\frac{1}{q_{l}}\left(\frac{\alpha_{l}^{m+1}\left(1-y_{l}^{n+1}\right)}{1-y_{l}}+P_{\mathrm{coll}, l}\left(1-\alpha_{l}^{m+1}\right) y_{l}^{n}\right. \\
& \left.\left.+\left(1-P_{\mathrm{coll}, l}\right) \frac{\left(1-\alpha_{l}^{m+1}\right)\left(1-y_{l}^{n+1}\right)}{1-y_{l}}\right)\right]^{-1},
\end{aligned}
$$

where $y_{l}=P_{\text {coll }, l}\left(1-\alpha_{l}^{m+1}\right)$. Further details on the derivation can be found in [14].

We derive the probability $\tau_{l}$ that node $V_{l}$ attempts CCA in a randomly chosen time unit as

$$
\tau_{l}=\sum_{i=0}^{m} \sum_{j=0}^{n} b_{i, 0, j}^{(l)}=\left(\frac{1-\alpha_{l}^{m+1}}{1-\alpha_{l}}\right)\left(\frac{1-y_{l}^{n+1}}{1-y_{l}}\right) b_{0,0,0}^{(l)} .
$$

This probability depends on the probability $\alpha_{l}$ that CCA is busy and the probability $P_{\text {coll }, l}$ that a transmitted packet encounters a collision. We study these probabilities next.

We derive the busy channel probability $\alpha_{l}$ as follows:

$$
\alpha_{l}=\alpha_{\mathrm{pkt}, l}+\alpha_{\mathrm{ack}, l},
$$

where $\alpha_{\mathrm{pkt}, l}$ is the probability that node $V_{l}$ senses the channel and finds it occupied by a packet transmission in the neighborhood $\Omega_{l}$, whereas $\alpha_{\text {ack }, l}$ is the probability of finding the channel busy due to ACK transmission from $V_{0}$.

The probability that node $V_{l}$ finds the channel busy due to a packet transmission is the combination of two events: (i) at least one node accesses the channel in one of the previous $L$ time units; (ii) at least one of the nodes that accessed the channel found it clear. We would like to remark here a major difference with the Markov chain model proposed in [5]. In homogeneous networks with no limitation in the sensing range, the busy channel probability is a network global information. In our case, it depends on the access and busy channel probabilities of each node in the neighborhood.

Denote by $\mathcal{S}_{l}$ the event that node $V_{l}$ is sensing, and by $\mathcal{T}_{l}$ the event that node $V_{l}$ is transmitting. Denote also by $\mathcal{F}_{l}$ the event that there is at least one transmission in $\Omega_{l}$. Then,

$$
\alpha_{\mathrm{pkt}, l}=\operatorname{Pr}\left[\mathcal{F}_{l} \mid \mathcal{S}_{l}\right]=\sum_{i=0}^{\left|\Omega_{l}\right|-2} \sum_{j=1}^{C_{l, i}} \operatorname{Pr}\left[\bigcup_{k=1}^{i+1} \mathcal{T}_{k_{j}} \mid \mathcal{S}_{l}\right],
$$

where $C_{l, i}=\left(\begin{array}{c}\left|\Omega_{l}\right|-1 \\ i+1\end{array}\right)$. The index $k$ accounts for the events of simultaneous transmissions in the channel and the index $j$ enumerates the combinations of events in which $i$ channel accesses are performed in the network simultaneously. Therefore, the index $k_{j}$ refers to the node in the $k$-th position 
in the $j$-th combination of $i$ elements out of $\Omega_{l}$, so that

$$
\alpha_{\mathrm{pkt}, l}=L \sum_{i=0}^{\left|\Omega_{l}\right|-2} \sum_{j=1}^{C_{l, i}} \prod_{k=1}^{i+1} \tau_{k_{j}}\left(1-\prod_{k=1}^{i+1} \alpha_{k_{j}}\right) \prod_{h=i+2}^{\left|\Omega_{l}\right|}\left(1-\tau_{h_{j}}\right) \text {. }
$$

To illustrate this formula, we use an example. Assume that there are two contending nodes in the neighborhood of $V_{4}$, e.g., $\Omega_{4}=\left\{V_{0}, V_{3}, V_{5}\right\}$ (see Fig. 1 1 ). Note that $V_{0}$ does not generate packets. Then, the event of busy channel for node $V_{4}$, is given by the sum of three contributions:

1) Only node $V_{3}$ accessed the channel and found it clear. The probability of this event is $L \tau_{3}\left(1-\tau_{5}\right)\left(1-\alpha_{3}\right)$.

2) Only node $V_{5}$ accessed the channel and found it clear. Similarly, the probability is $L \tau_{5}\left(1-\tau_{3}\right)\left(1-\alpha_{5}\right)$.

3 ) Both nodes accessed the channel and at least one node found it clear. Note that $V_{5}$ may not belong to $\Omega_{3}$. This probability is upper bounded by $L \tau_{3} \tau_{5}\left(1-\alpha_{3} \alpha_{5}\right)$.

Eq. (10) follows as a generalization of this example.

Let $R_{h}$ be the reliability on link $h$. A busy channel assessment due to ACKs depends on the probability of successful packet reception in $\Omega_{0}$. Therefore, we write $\alpha_{\text {ack, } l}$ as

$$
\alpha_{\text {ack }, l}=L_{\text {ack }} \sum_{h \in \Omega_{0} h \neq l} q_{h} R_{h}
$$

where $L_{\text {ack }}$ is the length of the ACK and $q_{h}$ is the packet generation rate of node $V_{h}$.

$P_{\text {coll }, l}$ is the probability that the packet transmission from node $V_{l}$ to the root node $V_{0}$ encounters a simultaneous packet transmission. There are three situations that determine a packet collision: (i) at least one node in $\Omega_{0}$ senses the channel in the same time slot as node $V_{l}$; (ii) at least one node in $\Omega_{0 \backslash l}$ (hidden node) has started a packet transmission in one of the previous $L$ backoff units; (iii) at least one node in $\Omega_{0 \backslash l}$ starts a packet transmission before node $V_{l}$ ends its transmission. We define by $\mathcal{A}_{l}$, the event of simultaneous sensing with node $V_{l}$ in $\Omega_{0}$, and by $\mathcal{B}_{l}$, the event of carrier sense failure, due to hidden terminals in $\Omega_{0 \backslash l}$. Therefore, the collision probability $P_{\text {coll }, l}$ is given by

$$
P_{\text {coll }, l}=\operatorname{Pr}\left[\mathcal{A}_{l}\right]+\left(1-\operatorname{Pr}\left(\mathcal{A}_{l}\right)\right) \operatorname{Pr}\left[\mathcal{B}_{l}\right] .
$$

If a generic node $V_{k}$ senses the channel with probability $\tau_{k}$, the event $\mathcal{A}_{l}$ occurs with probability

$$
\operatorname{Pr}\left[\mathcal{A}_{l}\right]=1-\prod_{k \in \Omega_{0} k \neq l}\left(1-\tau_{k}\right) .
$$

The probability of event $\mathcal{B}_{l}$ is equivalent to the busy channel probability in the set $\Omega_{0 \backslash l}$, hence,

$$
\begin{aligned}
\operatorname{Pr}\left[\mathcal{B}_{l}\right]=\sum_{i=0}^{\left|\Omega_{0 \backslash l}\right|-2} \sum_{j=1}^{C_{l, i}} \operatorname{Pr}\left[\bigcup_{k=1}^{i+1} \mathcal{T}_{k_{j}} \mid \mathcal{S}_{l}\right] \\
=2 L \sum_{i=0}^{\left|\Omega_{0 \backslash l}\right|-2} \sum_{j=1}^{C_{l, i}} \prod_{k=1}^{i+1} \tau_{k_{j}}\left(1-\prod_{k=1}^{i+1} \alpha_{k_{j}}\right) \prod_{h=i+2}^{\left|\Omega_{l}\right|}\left(1-\tau_{h_{j}}\right) .
\end{aligned}
$$

To complete the analysis, we need to derive an expression of the reliability for each link of the network. In slotted CSMA/CA, packets are discarded due to two reasons: (i) channel access failure or (ii) retry limits. Channel access failure happens when a packet fails to obtain clear channel within $m+1$ backoffs. Furthermore, a packet is discarded if the transmission fails due to repeated collisions after $n+1$ attempts. Following the Markov model presented in Fig. 2, the probability that the packet is discarded due to channel access failure is

$$
P_{\mathrm{cf}, l}=\frac{\alpha_{l}^{m+1}\left(1-\left(P_{\mathrm{coll}, l}\left(1-\alpha_{l}^{m+1}\right)\right)^{n+1}\right)}{1-P_{\mathrm{coll}, l}\left(1-\alpha_{l}^{m+1}\right)} .
$$

The probability of a packet discarded due to retry limits is

$$
P_{\mathrm{cr}, l}=\left(P_{\mathrm{coll}, l}\left(1-\alpha_{l}^{m+1}\right)\right)^{n+1} .
$$

Therefore, by using Eqs. (12) and (13), the reliability is

$$
R_{l}=1-\left(P_{\mathrm{cf}, l}+P_{\mathrm{cr}, l}\right) .
$$

The expressions of the carrier sensing probability $\tau_{l}$, the busy channel probability $\alpha_{l}$, and the reliability $R_{l}$, for $l=$ $1, \ldots, N$, form a system of non-linear equations that can be solved through numerical methods. The solution of these equations provides us with the performance analysis in terms of link reliability in single-hop networks. In the next subsection, we generalize the model to the multi-hop case.

\section{B. Extension to Multi-hop Networks}

In a multi-hop topology, the number of links is not equal to the number of nodes. We associate to each link $l$, a pair transmitter-receiver $\left(V_{i}, V_{j}\right)$. The proposed Markov chain model can be easily extended to a generic network in which information is routed through multi-hop communications to the root node. The Markov chain model should be solved for each link of the network, by considering now that the generic destination node $V_{j}$ in each link has a different neighborhood $\Omega_{j}$, and generates its own traffic $\lambda_{j}$.

Let $\lambda=\left[0, \lambda_{1}, \ldots, \lambda_{N}\right]$ be a vector of traffic generation rates, where each component is associated to a node. In addition, every node has to forward traffic generated by nodes in its children set $\Delta_{l}$. The scope of the following analysis is to provide an expression of the total traffic load in each node, which we associate to the probability $q_{l}$ in the per-link Markov chain in Fig. 2. To do so, we must characterize the traffic distribution in the network according to the routing policy.

Let $\pi_{i, j}$ be the metric associated to link $\left(V_{i}, V_{j}\right)$ to build the routing graph, as specified by ROLL [2]. At the routing layer, metrics are chosen to be static if the network is stationary. Anyhow, due to the dynamic nature of wireless connectivity, link attributes including reliability may change over time and the routing metrics are updated accordingly. We can represent this dynamical behavior using a statistical analysis. The effect of routing can be described by a matrix $\boldsymbol{T} \in \mathbb{R}^{(N+1) \times(N+1)}$, in which the generic element $T_{i, j}$ corresponds to the probability that the metric in the link $l=\left(V_{i}, V_{j}\right)$ is the highest among the set of candidate receivers $\Gamma_{i}$, i.e.,

$$
T_{i, j}=\operatorname{Pr}\left[\pi_{i, j}=\max _{V_{h} \in \Gamma_{i}} \pi_{i, h}\right] .
$$

The distribution of the traffic flows along the network can be represented by the matrix $\boldsymbol{T}$, opportunely scaled by the probability of successful reception in each link. Therefore, we 
define a matrix $\widetilde{\boldsymbol{T}}$ such that $\widetilde{T}_{i, j}=T_{i, j} R_{l}$ where $R_{l}$ is the reliability in the link $l=\left(V_{i}, V_{j}\right)$. The vector of node traffic generation probabilities $Q=\left[0, q_{1}, \ldots, q_{N}\right]$ is the solution of a system of flow balance equations:

$$
Q=Q \widetilde{\mathbf{T}}+\lambda .
$$

Note that $\lambda$ must be scaled by aUnitBackoffPeriod (see Sec. (III). In steady state conditions, we have

$$
Q=\lambda[\mathbf{I}-\widetilde{\mathbf{T}}]^{-1} .
$$

where $\boldsymbol{I}$ is the identity matrix $\boldsymbol{I} \in \mathbb{R}^{(N+1) \times(N+1)}$. Eq. (15) gives the fundamental relation between the idle packet generation probability $q_{l}$, the effect of routing (through the matrix T) and the performance at MAC layer (through the link reliability $R_{l}$ ). To obtain the solution of the model for multihop network we couple Eq. (15), with the expressions for $\tau_{l}$, $\alpha_{l}$ and $R_{l}$, as obtained by Eqs. (8), (9), and (14). Note that we now refer to link $l$ as the link between the pair transmitterreceiver $\left(V_{i}, V_{j}\right)$, and the notation in the derivation changes accordingly. Furthermore, in the derivation of the busy channel probability in multi-hop networks due to ACK transmission $\alpha_{\text {ack }, l}$, we replace Eq (11) with Eq. (16),

$$
\alpha_{\text {ack }, l}=L_{\text {ack }} \sum_{j \in \Omega_{i}} \sum_{h \in \Delta_{j}} q_{h} \widetilde{T}_{i, j},
$$

which includes the effect of limited carrier sensing range at the destination and the routing matrix $\boldsymbol{T}$. Recall $\Delta_{j}$ is the set of transmitters that have node $V_{j}$ as destination (children nodes).

The solution of the analysis provides us with per-link reliability in the network. We derive the end-to-end reliability of $V_{i}$ by the product of link radiabilities in the path to $V_{0}$.

\section{RESUlTs}

In this section, we present extensive Monte Carlo simulations of unslotted IEEE 802.15.4 to validate our analysis. The simulations are based on the specifications of the IEEE 802.15.4 [1] with several values of the traffic condition and node sensing range. We set the default MAC parameters to $m_{0}=3, m=4, m_{b}=7, n=0$. Other settings give similar results to those discussed next. First, we consider single-hop networks and then multi-hop topologies.

\section{A. Single-hop Topology}

In this first set of simulation results, we validate the model proposed in Sec. III-A for a single-hop topology (e.g., Fig. 1h).

We consider two basic scenarios to study the impact of hidden terminals, namely $\left|\Omega_{l}\right|=N$, which is denoted by full sensing capability, and $\left|\Omega_{l}\right|=3$ which represents a condition of reduced sensing capability (the neighborhood is composed by the root node $V_{0}$ and two adjacent nodes).

Fig. 3 shows the average reliability computed over all the links for a single-hop network with homogeneous traffic. On the $\mathrm{x}$-axis the node packet generation rate is reported. Results are shown for different sizes of the network $(N=7, N=14)$, and by considering both full and reduced sensing capability. As a reference, we report the reliability obtained from the single-hop homogeneous model with full sensing capabilities, presented in [5] and readapted to the unslotted mechanism of IEEE 802.15.4. A good agreement between simulations and analytical model results is obtained. The proposed model performance with full sensing capabilities coincides with the unslotted version of [5]. There is a small gap between the two models for high traffic and number of nodes, due to the different assumption in the derivation of the busy channel probability, as remarked in Sec. III. By reducing the carrier sensing capabilities, a critical impact on the reliability is observed, which cannot be predicted by the model in [5].

Consider the heterogeneous traffic and the combined effect with reduced sensing capabilities. In Fig. 4, we report the link reliability associated to each node. We plot analytical results and simulation of the reliability for a single-hop network with $N=7$ nodes. In the homogeneous case, each node generates the same traffic $\lambda_{l}=5 \mathrm{pkt} / \mathrm{s}, l=1, \ldots, N$. In the heterogeneous case, node $V_{4}$ generates a traffic $\lambda_{4}=20 \mathrm{pkt} / \mathrm{s}$, while the rest of the networks operates with $\lambda_{l}=5 \mathrm{pkt} / \mathrm{s}$. The effect of an increasing traffic in $V_{4}$ leads to a decreasing of the reliability in the rest of the network, whereas the reliability of $V_{4}$ is only marginally affected. This effect is more significant when there are reduced sensing capabilities. In the next section, we show how the relation between traffic, carrier sensing range and reliability can interact with the routing in multi-hop networks.

\section{B. Multi-hop Topology}

In this section, we validate the reliability analysis for multihop networks proposed in Sec. III-B. We consider the topology of Fig. $1 \mathrm{~b}$. However, the results we present are general and can be applied to every topology. We focus on two cases, which we denote by Path 1 and Path 2. In Path 1, we analyze the endto-end reliability from node $V_{7}$, which routes its packets along the path $V_{4}-V_{1}$ to the root node $V_{0}$. In Path 2, $V_{7}$ forwards its packets along the path $V_{6}-V_{3}$. We also distinguish between coupled and isolated paths. Coupling is obtained by letting the carrier sensing range of nodes in a path include nodes in the other path, up to two hops away. In the isolated case, nodes along the two paths do not sense each other, except for $V_{0}$.

In Fig. 5, we report the end-to-end reliability from node $V_{7}$ to the root node $V_{0}$, by varying the node packet generation rate, when the two paths are isolated. From both analytical results and simulations, Path 2 outperforms Path 1 in terms of reliability and the difference increases as the traffic increases.

Fig. 6 shows the end-to-end reliability for analytical model and simulations of the multi-hop network with coupled paths. Compared to Fig. 5, the result is different and the best performance in terms of reliability is on Path 1. The reason is that the dominant nodes in terms of traffic load affect negatively the performance of the nodes connected to the same destination, as shown for the single-hop topology. We recall that the reliability of a contention access scheme increases as the average number of contenders in each time unit reduces. The natural consequence is that, if the routing metric is based only on the maximization of the end-to-end reliability, and there is strong coupling in the network, the routing decision leads to unbalanced distribution of traffic load, by forcing the forwarded traffic to dominant nodes. If not taken in account, this phenomenon may cause stability issues when considering limited node buffer size and energy constraints. 


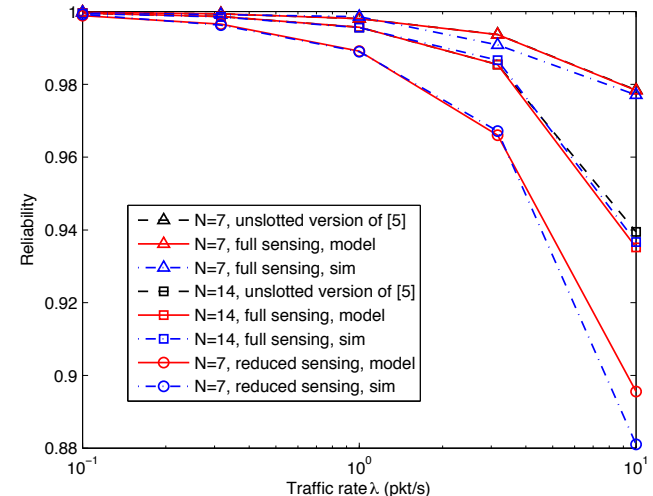

Fig. 3. Single-hop topology in Fig.11: reliability vs traffic rate $\lambda$. Full sensing and reduced sensing corresponds to $\left|\Omega_{l}\right|=N$ and $\left|\Omega_{l}\right|=3$, respectively. Unslotted version of [5] refers to the single-hop homogeneous model in [5], by adapting the analysis to the unslotted mechanism of IEEE 802.15.4. Note that for $N=7$ and full sensing the curve of the proposed model coincides with the unslotted version of [5].

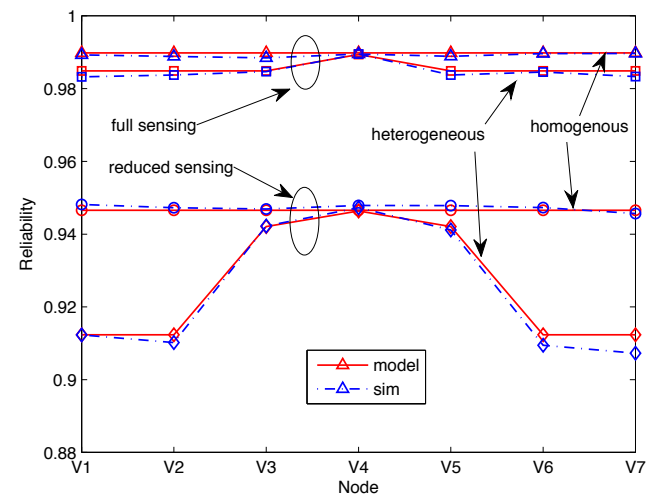

Fig. 4. Single-hop topology in Fig. 1h: node reliability. Full sensing and reduced sensing corresponds to $\left|\Omega_{l}\right|=N$ and $\left|\Omega_{l}\right|=3$, respectively. In homogeneous case, $\lambda_{l}=5 \mathrm{pkt} / \mathrm{s}$, for $l=1, \ldots, N$. In heterogeneous case, $\lambda_{4}=20 \mathrm{pkt} / \mathrm{s}, \lambda_{l}=5 \mathrm{pkt} / \mathrm{s}$, for $l \neq 4$.

We remark that our results abstracts from the channel behavior. The goal is not to provide a routing solution but to show the effectiveness of the model in capturing the interaction between IEEE 802.15.4 MAC performance and routing decisions.

\section{Conclusions}

In this paper we proposed a framework for the analysis of multi-hop networks using the unslotted IEEE 802.15.4 MAC. We extended the Markov chain analysis originally proposed by Bianchi, to include important features of multi-hop networks, as heterogeneous distribution of the traffic and hidden terminal nodes. We showed the mutual influence between routing decisions and MAC performance in terms of reliability. Future works include an extensive study of the interaction between various routing metrics and MAC performance indicators.

\section{REFERENCES}

[1] IEEE 802.15.4 Wireless Medium Access Control (MAC) and Physical Layer (PHY) Specifications for Low-Rate Wireless Personal Area Networks (WPANs), 2006. [Online]. Available: http://www.ieee802.org/15

[2] Routing over low power and lossy networks (ROLL). [Online]. Available: http://www.ietf.org/dyn/wg/charter/roll-charter.html

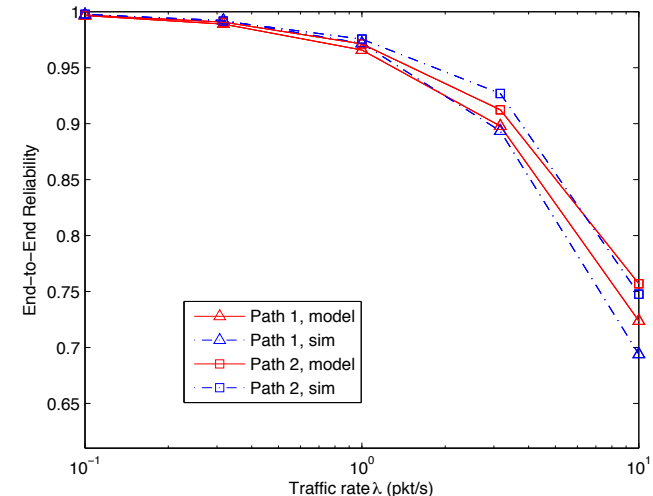

Fig. 5. Multi-hop topology in Fig. 1b: end-to-end reliability for isolated paths. Path 1 is $V_{7}-V_{4}-V_{1}-V_{0}$, when the link $V_{7}-V_{6}$ is disabled. Path 2 is $V_{7}-V_{6}-V_{3}-V_{0}$, when the link $V_{7}-V_{4}$ is disabled. Notice the different scale on the $y$ axis compared to the link reliability in Figs. 3 and 4

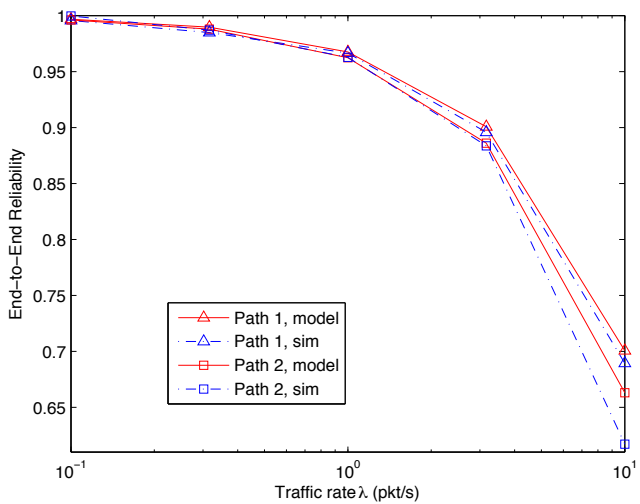

Fig. 6. Multi-hop topology in Fig. 1b: end-to-end reliability for coupled paths. Path 1 is $V_{7}-V_{4}-V_{1}-V_{0}$, when the link $V_{7}-V_{6}$ is disabled. Path 2 is $V_{7}-V_{6}-V_{3}-V_{0}$, when the link $V_{7}-V_{4}$ is disabled.

[3] S. Pollin, M. Ergen, S. C. Ergen, B. Bougard, F. Catthoor, A. Bahai, and P. Varaiya, "Performance analysis of slotted carrier sense IEEE 802.15.4 acknowledged uplink transmissions," in IEEE WCNC, 2008.

[4] J. Mišić, S. Shaf, and V. Mišić, "Performance of a beacon enabled IEEE 802.15.4 cluster with downlink and uplink traffic," IEEE Transactions Parallel and Distributed Systems, pp. 361-376, April 2006.

[5] P. Park, P. Di Marco, P. Soldati, C. Fischione, and K. H. Johansson, "A generalized Markov chain model for effective analysis of slotted IEEE 802.15.4," in IEEE MASS, 2009.

[6] G. Bianchi, "Performance analysis of the IEEE 802.11 distributed cordination function," IEEE Journal on Selected Areas in Communications, pp. 535 - 547, March 2000.

[7] D. Malone, K. Duffy, and D. Leith, "Modeling the 802.11 distributed coordination function in nonsaturated heterogeneous conditions," IEEE Transactions on Networking, pp. 159-172, February 2007.

[8] M. Chen, G. Liu, D. Wu, and G. Zhu, "A unified model for performance analysis of 802.11 in heterogeneous traffic and saturation condition," in IEEE ICC, 2008.

[9] E. Ndih, N. Khaled, and G. De Micheli, "An analytical model for the contention access period of the slotted IEEE 802.15.4 with service differentiation," in IEEE ICC, 2009.

[10] T. Kim and J. Lim, "Throughput analysis considering coupling effect in IEEE 802.11 networks with hidden stations," IEEE Communication Letters, pp. 175-177, March 2009.

[11] H. Wu, F. Zhu, Q. Zhang, and Z. Niu, "Analysis of IEEE 802.11 DCF with hidden terminals," in IEEE Globecom, 2006.

[12] M. Hira, F. Tobagi, and K. Medepalli, "Throughput analysis of a path in an IEEE 802.11 multihop wireless network," in IEEE WCNC, 2007.

[13] J. Baras, V. Tabatabaee, P. Papageorgiou, and N. Rentz, "Modelling and optimization for multi-hop wireless networks using fixed point and automatic differentiation," in WiOpt, 2008.

[14] P. Di Marco, P. Park, C. Fischione, and K. H. Johansson, "Analytical model of multi-hop IEEE 802.15.4 networks," Royal Institute of Technology, Tech. Rep., March 2010. 